\title{
Equilibria of Multibody Chain in Orbit Plane
}

\author{
Anna D. Guerman* \\ University of Beira Interior, 6201-001 Covilhã, Portugal
}

\begin{abstract}
We study equilibria of a multibody system in the orbit plane within the framework of a model of $n+1$ material points connected by $n$ light rods into an $n$-link chain. The junctions are spherical hinges. The center of mass of the system moves along a circular orbit. The equilibrium equations are reduced to a fairly simple form that enables their analysis. We find all the equilibria of an $\boldsymbol{n}$-link chain in the orbit plane and prove that each rod can occupy one of the following three positions: it can be directed along the tangent to the orbit of the center of mass of the chain; it can be a member of a group of adjacent vertical rods, being the center of mass of this group situated on the tangent to the orbit; and, finally, an oblique orientation is possible if the rod joins either two vertical groups of rods or the end of a vertical group with the tangent to the orbit. It is shown that the number of equilibria does not exceed $2^{2 n}$. We include the analysis of two examples (three- and four-link chains) and represent the schemes of all the realizable equilibria in these cases.
\end{abstract}

\section{Introduction}

$\mathbf{O}$ RBITAL dynamics of a system of connected bodies is of great interest due to its numerous possible applications, including orbital stations, robots, tethered systems with multiple payloads, and formation flying. The first studies on the subject were published in the early 1960s and were followed by much scientific research and many engineering projects. A large part of this work is dedicated to the behavior of two tethered bodies. However, there exists significant interest for multibody systems, inspired by various space programs under development. The study of multibody systems is mainly focused on numerical analysis (see, for instance, Refs. 1-4). Analyticalresearchis mostly restrictedeither to systems with a small number of connected bodies (see Refs. 5-9 for chains with two or three bodies) or to systems with specific additional conditions (such as the orbiting ring studied in Refs. 10 and 11).

In this paper, we suggest an analytical study of a system that includes an arbitrary number of satellites joined into an open chain by light rigid rods. We impose no restrictions on the masses of satellites or the lengths of the links. We model the satellites by material points and suppose that the mass of the links is negligible compared with the masses of the satellites. (This approach is used in most analytical studies of tethered systems, such as Refs. 6-9 and 11.) We study equilibrium configurations of this system in the orbital reference frame.

Some particularcases of this problem were examined previously. The two-member chain (double pendulum) is a particular case of two connected bodies whose in-plane equilibria were studied in Ref. 5. The model we consider was used ${ }^{7-9}$ to study equilibria of three linked material points. It was shown that there exist only three kinds of in-plane configurations: both rods directed along the local vertical; both rods aligned with the tangent to the orbit of the center of masses; and one rod vertical while the other is in an oblique position. The in-plane equilibria of a two-link chain were studied, ${ }^{7}$ and the principal frequencies of small-amplitude motions about these equilibria were determined. All the spatial equilibria of a double pendulum were found in Ref. 8. A study of equilibria of a double pendulum in the plane of a circular orbit was performed in Ref. 9, and stability of all the in-plane configurations was analyzed.

Presented as Paper AAS 02-169 at the AAS/AIAA 12th Space Flight Mechanics Meeting, San Antonio, TX, January 2002; received 7 June 2002; revision received 2 April 2003; accepted for publication 8 May 2003. Copyright (C) 2003 by Anna D. Guerman. Published by the American Institute of Aeronautics and Astronautics, Inc., with permission. Copies of this paper may be made for personal or internal use, on condition that the copier pay the $\$ 10.00$ per-copy fee to the Copyright Clearance Center, Inc., 222 Rosewood Drive, Danvers, MA 01923; include the code 0731-5090/03 \$10.00 in correspondence with the CCC.

*Assistant Professor, Department of Electromechanical Engineering; Anna@demnet.ubi.pt. Member AIAA.

\section{Posing the Problem}

Let us consider a system of $n+1$ material points, $A_{0}, A_{1}, \ldots, A_{n}$, with respective masses $m_{0}, m_{1}, \ldots, m_{n}$. These points are connected by $n$ light rigid $\operatorname{rods}\left(a_{k}=A_{k-1} A_{k}, k=1, \ldots, n\right)$ into an open chain. The junctions are spherical hinges. The gravitational field of the Earth is supposed to be central Newtonian. The center of mass of the system $O$ moves along a circular orbit with angular velocity $\omega$.

To describe the behavior of this system we use the orbital reference frame $O x y z$. Its $z$ axis follows the local vertical, the $x$ axis is tangent to the orbit and points in the direction of the velocity of point $O$, and so the $y$ axis is normal to the plane of the orbit. The orientation of the $\operatorname{rod} a_{k}=A_{k-1} A_{k}$ is described by the angle $\phi_{k}$ between the rod $a_{k}$ and the $z$ axis (Fig. 1).

The components of the vector $\boldsymbol{a}_{k}=\boldsymbol{A}_{k-1} \boldsymbol{A}_{k}$ are

$$
\begin{gathered}
a_{k x}=\xi_{k}=a_{k} \sin \phi_{k}, \quad a_{k y}=\eta_{k}=0 \\
a_{k z}=\zeta_{k}=a_{k} \cos \phi_{k}
\end{gathered}
$$

The coordinates of the point $A_{k}(k=1, \ldots, n)$ in the orbital frame are

$$
x_{k}=x_{0}+\sum_{s=1}^{k} \xi_{s}, \quad y_{k}=0, \quad z_{k}=z_{0}+\sum_{s=1}^{k} \zeta_{s}
$$

where $x_{0}, y_{0}$, and $z_{0}$ are the coordinates of the first satellite in the chain $A_{0}$. We denote by $x_{*}, y_{*}$, and $z_{*}$ the coordinates of the center of mass $O$ of the structure. For this choice of the reference frame, $x_{*}=y_{*}=z_{*}=0$, and we obtain

$$
\sum_{k=0}^{n} m_{k} x_{k}=M x_{*}=0, \quad \sum_{k=0}^{n} m_{k} z_{k}=M z_{*}=0
$$

Then one can determine the position of the point $A_{0}$ :

$$
x_{0}=-\frac{1}{M} \sum_{s=1}^{n} M_{s}^{n} \xi_{s}, \quad z_{0}=-\frac{1}{M} \sum_{s=1}^{n} M_{s}^{n} \zeta_{s}
$$

We use the notation

$$
M_{i}^{j}=\sum_{p=i}^{j} m_{p}, \quad M_{0}^{n}=M
$$

(the total mass of the chain). Thus, the system considered has $n$ degrees of freedom, and $\phi_{k}(k=1, \ldots, n)$ represent the generalized coordinates.

The kinetic energy $T$ of the system is

$$
T=\frac{1}{2} \sum_{k=0}^{n} m_{k}\left\{\left[\dot{x}_{k}+\omega\left(z_{k}+r_{O}\right)\right]^{2}+\left(\dot{z}_{k}-\omega x_{k}\right)^{2}\right\}
$$




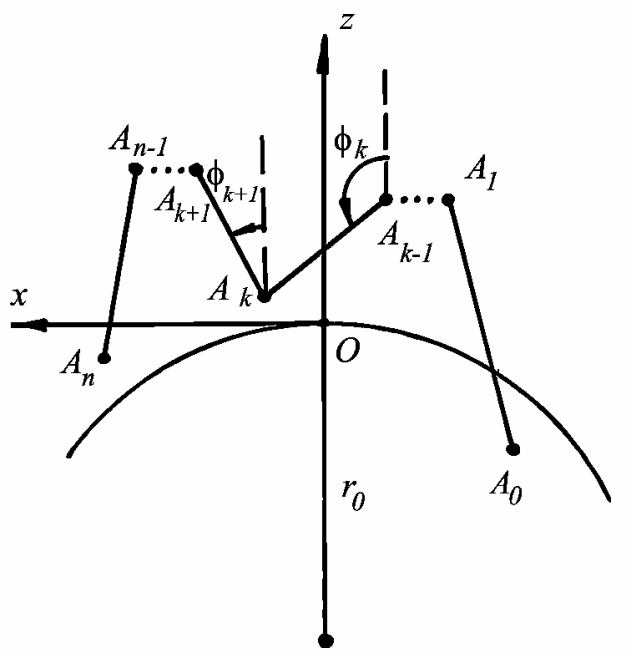

Fig. 1 Orientation angles.

Its gravitational potential energy is

$$
V=-\omega^{2} r_{o}^{3} \sum_{k=0}^{n} \frac{m_{k}}{r_{k}}
$$

where $r_{k}$ is the distance between the point $A_{k}$ and the center of the Earth,

$$
r_{k}=\sqrt{x_{k}^{2}+\left(r_{O}+z_{k}\right)^{2}}
$$

and $r_{O}$ represents this distance for the center of mass $O$ of the system (that is, $r_{O}$ is the radius of the orbit). Calculating the function $V$ and taking into account the terms up to the second order of $a_{k} / r_{O}$, one gets

$$
\begin{aligned}
V= & -\omega^{2} r_{O}^{3} \sum_{k=0}^{n} m_{k}\left[x_{k}^{2}+\left(r_{O}+z_{k}\right)^{2}\right]^{-\frac{1}{2}} \\
& =-\omega^{2} r_{O}^{2} \sum_{k=0}^{n} m_{k}\left(1+2 \frac{z_{k}}{r_{O}}+\frac{x_{k}^{2}+z_{k}^{2}}{r_{O}^{2}}\right)^{-\frac{1}{2}} \\
& =-\omega^{2} r_{O}^{2} \sum_{k=0}^{n} m_{k}\left(1-\frac{z_{k}}{r_{O}}-\frac{x_{k}^{2}-2 z_{k}^{2}}{r_{O}^{2}}\right) \\
& =-M \omega^{2} r_{O}^{2}+\frac{\omega^{2}}{2} \sum_{k=0}^{n} m_{k}\left(x_{k}^{2}-2 z_{k}^{2}\right)
\end{aligned}
$$

[We used relation (3) once more.]

\section{Equations of Equilibria}

Equilibria of the chain correspond to the solutions

$$
\phi_{k}=\phi_{k 0}=\mathrm{const}
$$

of the equations

$$
\frac{\partial\left(T_{0}-V\right)}{\partial \phi_{k}}=0, \quad k=1, \ldots, n
$$

where $T_{0}$ represents the part of $T$ independent of the velocities, and $T_{0}-V$ is sometimes referred to as the dynamic potential. Simplifying the term $T_{0}$ using relation (3), one gets

$$
T_{0}-V=\frac{3 \omega^{2}}{2} \sum_{k=0}^{n} m_{k} z_{k}^{2}+\mathrm{const}
$$

and so the in-plane equilibrium configurations of the chain are described by the following equations:

$$
\sum_{k=0}^{n} m_{k} z_{k} \frac{\partial z_{k}}{\partial \phi_{p}}=0, \quad p=1, \ldots, n
$$

Substituting Eq. (4) into system (2), we arrive at

$$
z_{k}=\frac{1}{M}\left(\sum_{s=1}^{k} M_{0}^{s-1} \zeta_{s}-\sum_{s=k+1}^{n} M_{s}^{n} \zeta_{s}\right), \quad 0 \leq k \leq n
$$

For the sake of uniformity we assume the convention that

$$
\sum_{s=1}^{0} M_{0}^{s-1} \zeta_{s}=0, \quad \sum_{s=n+1}^{n} M_{s}^{n} \zeta_{s}=0
$$

So

$$
\frac{\partial z_{k}}{\partial \phi_{p}}= \begin{cases}\frac{1}{M} M_{p}^{n} a_{p} \sin \phi_{p}, & \text { for } \quad k<p, \quad 0 \leq k, p \leq n \\ -\frac{1}{M} M_{0}^{p-1} a_{p} \sin \phi_{p}, & \text { for } \quad k \geq p\end{cases}
$$

and rather tedious calculations lead to the following:

$$
\begin{aligned}
\sum_{k=0}^{n} m_{k} z_{k} \frac{\partial z_{k}}{\partial \phi_{p}}=\left[\sum_{k=0}^{p-1} \frac{1}{M^{2}} m_{k}\left(\sum_{s=1}^{k} M_{0}^{s-1} \zeta_{s}-\sum_{s=k+1}^{n} M_{s}^{n} \zeta_{s}\right) M_{p}^{n}\right. \\
\left.-\sum_{k=p}^{n} \frac{1}{M^{2}} m_{k}\left(\sum_{s=1}^{k} M_{0}^{s-1} \zeta_{s}-\sum_{s=k+1}^{n} M_{s}^{n} \zeta_{s}\right) M_{0}^{p-1}\right] a_{p} \sin \phi_{p} \\
=\frac{a_{p} \sin \phi_{p}}{M^{2}}\left[M _ { p } ^ { n } \left(\sum_{s=1}^{p-1} M_{0}^{s-1} M_{s}^{p-1} \zeta_{s}-\sum_{s=1}^{p} M_{0}^{s-1} M_{s}^{n} \zeta_{s}\right.\right. \\
\left.-\sum_{s=p+1}^{n} M_{0}^{p-1} M_{s}^{n} \zeta_{s}\right)+M_{0}^{p-1}\left(\sum_{s=p+1}^{n} M_{p}^{s-1} M_{s}^{n} \zeta_{s}\right. \\
\left.\left.-\sum_{s=1}^{p} M_{0}^{s-1} M_{p}^{n} \zeta_{s}-\sum_{s=p+1}^{n} M_{0}^{s-1} M_{s}^{n} \zeta_{s}\right)\right] \\
=-\frac{a_{p} \sin \phi_{p}}{M}\left(M_{p}^{n} \sum_{s=1}^{p} M_{0}^{s-1} \zeta_{s}+M_{0}^{p-1} \sum_{s=p+1}^{n} M_{s}^{n} \zeta_{s}\right)
\end{aligned}
$$

Finally we arrive at the following system:

$$
\sin \phi_{k} \cdot\left(\boldsymbol{b}_{k} \boldsymbol{z}\right)=0, \quad k=1, \ldots, n
$$

Here $z=\left(\zeta_{1}, \ldots, \zeta_{n}\right)^{T}$,

$\boldsymbol{b}_{1}=\left(1 / M^{2}\right)\left(m_{0} M_{1}^{n}, m_{0} M_{2}^{n}, \ldots, m_{0}\left(m_{n-1}+m_{n}\right), m_{0} m_{n}\right)$

$\boldsymbol{b}_{n}=\left(1 / M^{2}\right)\left[m_{0} m_{n},\left(m_{0}+m_{1}\right) m_{n}, \ldots, M_{0}^{n-2} m_{n}, M_{0}^{n-1} m_{n}\right]$

$\boldsymbol{b}_{k}=\left(1 / M^{2}\right)\left[m_{0} M_{k}^{n},\left(m_{0}+m_{1}\right) M_{k}^{n}, \ldots, M_{0}^{k-2} M_{k}^{n}, M_{0}^{k-1} M_{k}^{n}\right.$,

$$
\left.M_{0}^{k-1} M_{k+1}^{n}, \ldots, M_{0}^{k-1} m_{n}\right], \quad 2 \leq k \leq n-1
$$

Here $b_{k}$ is the row $k$ of the matrix $\mathbb{B}\left(n ; m_{0}, \ldots, m_{n}\right)$ with the element in row $i$ and column $j$ equal to

$$
b_{i, j}=\left\{\begin{array}{lll}
\frac{1}{M^{2}} M_{0}^{j-1} M_{i}^{n}, & \text { for } & j \leq i \\
\frac{1}{M^{2}} M_{0}^{i-1} M_{j}^{n}, & \text { for } & j<i
\end{array}\right.
$$

The matrix $\mathbb{B}$ will be referred to as the mass matrix. 


\section{Properties of the Mass Matrix}

We now describe some important properties of the matrix $\mathbb{B}$. They will be our main tool in the analysis of system (16). These properties permit us to identify some elementary equilibrium configurations and then to describe all possible equilibria using superposition.

In the following analysis, we denote by $\mathbb{D}_{J}$ the matrix obtained from $\mathbb{B}$ by exclusion of rows and columns with numbers in the set $J: \mathbb{D}_{J}=\left(b_{i, j} \mid i, j \notin J\right) ; \boldsymbol{d}_{k J}$ stands for the $k$ th column of $\mathbb{B}$ without elements with indices from $J: \boldsymbol{d}_{k J}=\left(b_{j, k} \mid j \notin J\right)^{T}$ and $z_{J}$ stands for the column $z$ without these elements: $z_{J}=\left(\zeta_{j} \mid j \notin J\right)^{T}$.

Property 1: The neighboring rows of this matrix satisfy the relations

$$
\begin{gathered}
\left(M / m_{k-1}\right)\left(\boldsymbol{b}_{k}-\boldsymbol{b}_{k-1}\right)+\left(M / m_{k}\right)\left(\boldsymbol{b}_{k}-\boldsymbol{b}_{k+1}\right)=\boldsymbol{e}_{k} \\
k=2, \ldots, n-1 \\
\left(M / m_{0}\right) \boldsymbol{b}_{1}+\left(M / m_{1}\right)\left(\boldsymbol{b}_{1}-\boldsymbol{b}_{2}\right)=\boldsymbol{e}_{1} \\
\left(M / m_{n-1}\right)\left(\boldsymbol{b}_{n}-\boldsymbol{b}_{n-1}\right)+\left(M / m_{n}\right) \boldsymbol{b}_{n}=\boldsymbol{e}_{n}
\end{gathered}
$$

where $\boldsymbol{e}_{k}=\left(\delta_{k 1}, \ldots, \delta_{k n}\right)$ designates the $k$ th row of the identity matrix $n \times n\left(\delta_{k j}\right.$ is the Kronecker symbol).

Property 2: For $J=\{k+1, \ldots, k+r\}, k \geq 1, k+r \leq n$, the matrix $\mathbb{D}_{J}$ coincides with $\mathbb{B}$ for the $(n-r)$-link chain with the masses $\tilde{m}_{j}=m_{j}, j=1, \ldots, k-1 ; \tilde{m}_{k}=M_{k}^{k+r}, \tilde{m}_{j}=m_{j+r}$, $j=k+1, \ldots, n-r$ :

$$
\mathbb{D}_{J}=\mathbb{B}\left(n-r ; m_{0}, \ldots, m_{k-1}, M_{k}^{k+r}, m_{k+r+1}, \ldots, m_{n}\right)
$$

In the particular case $J=\{1, \ldots, r\}, \mathbb{D}_{J}$ coincides with the mass matrix $\mathbb{B}$ for the $(n-r)$-link chain with the masses $\tilde{m}_{1}=M_{0}^{r}$, $\tilde{m}_{j}=m_{j+r}, j=2, \ldots, n-r$.

Properties 1 and 2 can be proved by direct substitution.

Property 3: The matrix $\mathbb{B}$ does not degenerate:

$$
\operatorname{det} \mathbb{B}=\left(m_{0} m_{1}, \ldots, m_{n}\right) / M^{n+1} \neq 0
$$

We prove this equality by induction

For $n=1$, one gets $\mathbb{B}=m_{0} m_{1} / M^{2}$, and the statement is true.

Now we suppose that Eq. (22) is valid for $n=k-1$ and prove it for $n=k$. For the latter case we apply Eq. (20) to the last row of $\mathbb{B}$ and get

$$
\operatorname{det} \mathbb{B}=\frac{m_{k-1} m_{k}}{M\left(m_{k-1}+m_{k}\right)} \operatorname{det} \mathbb{D}_{J}
$$

where $J=\{n\}$. In accordance with Eq. (21), $\mathbb{D}_{J}=\mathbb{B}\left(k-1 ; m_{0}, \ldots\right.$, $m_{k-2}, m_{k-1}+m_{k}$ ), and we can use Eq. (22):

$$
\operatorname{det} \mathbb{D}_{J}=\left[m_{0} m_{1}, \ldots, m_{k-2}\left(m_{k-1}+m_{k}\right)\right] / M^{k}
$$

Finally,

$$
\begin{aligned}
\operatorname{det} \mathbb{B} & =\frac{m_{k-1} m_{k}}{M\left(m_{k-1}+m_{k}\right)} \frac{m_{0} m_{1}, \ldots, m_{k-2}\left(m_{k-1}+m_{k}\right)}{M^{k}} \\
& =\frac{\prod_{j=0}^{k} m_{j}}{M^{k+1}}
\end{aligned}
$$

which proves equality (22) for an $n$-link chain.

Property 4: For an arbitrary set $J$, the matrix $\mathbb{D}_{J}$ does not degenerate either:

$$
\operatorname{det} \mathbb{D}_{J} \neq 0
$$

Suppose that $J=\bigcup_{s} J_{s}$, where $J_{s}=\left\{k_{s}+1, \ldots, k_{s}+r_{s}\right\}$. Applying Property $2 s$ times, one concludes that $\mathbb{D}_{J}$ coincides with the matrix $\mathbb{B}$ for a $p$-link chain $\left(p=n-\sum_{s} r_{s}\right)$ with masses $\tilde{m}_{j}=m_{j}$, $j \notin J_{1}=J \bigcup\left(\bigcup_{s} k_{s}\right), \tilde{m}_{k_{s}}=M_{k_{s}}^{k_{s}+r_{s}}$. Thus,

$$
\operatorname{det} \mathbb{D}_{J}=\frac{\prod \tilde{m}_{j}}{M^{p+1}} \neq 0
$$

\section{Equilibrium Configurations}

In this section, we describe all of the possible solutions of system (16) and the corresponding configurations of a chain with an arbitrary number of satellites. These general results will later be illustrated in Sec. VI by two particular cases: equilibria of a threelink chain with different masses of satellites and lengths of the links (Fig. 2) and equilibria of a four-link chain with equal masses of points and lengths of rods (Figs. 3-6).

In Eqs. (16), both factors can vanish independently. Thus, to describe all the equilibria we have to study all these possibilities

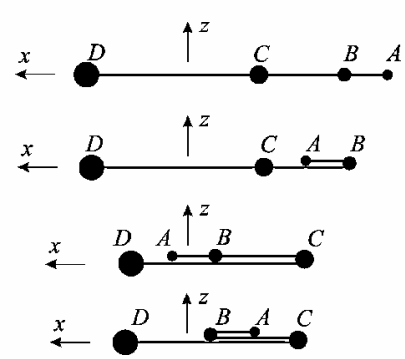

a)

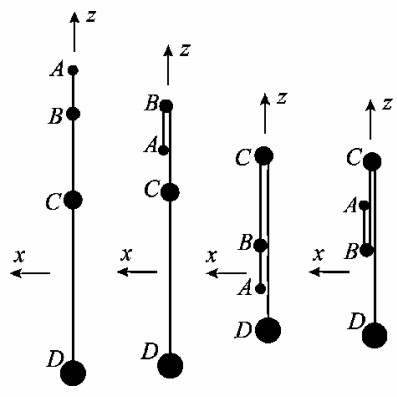

d)
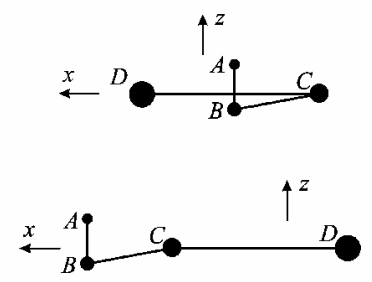

b)

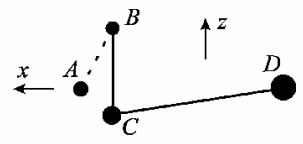

e)

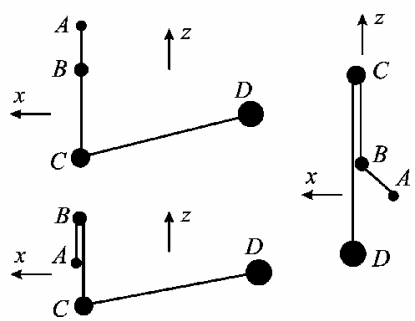

c)

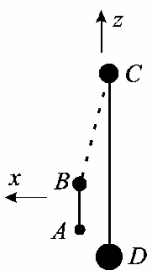

f)

Fig. 2 Study of a three-link chain: a-d) existing equilibrium configurations and $e$ and $f$ ) equilibria cannot be realized.

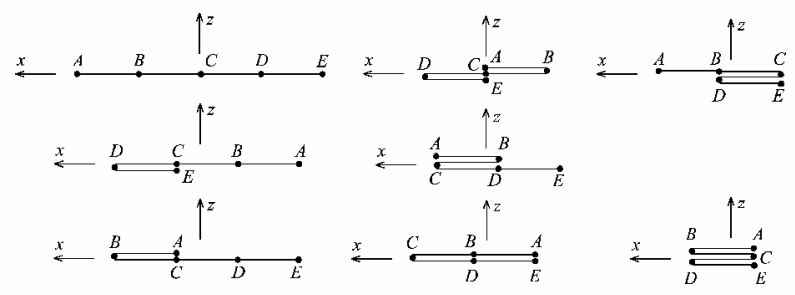

a)

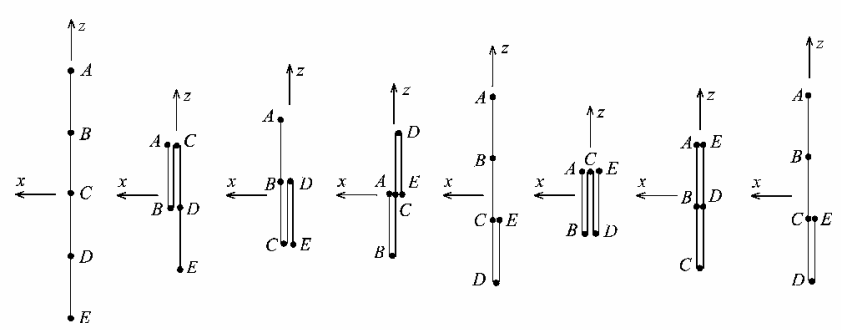

b)

Fig. 3 Equilibria of a four-link chain: a) horizontal and b) vertical configurations. 


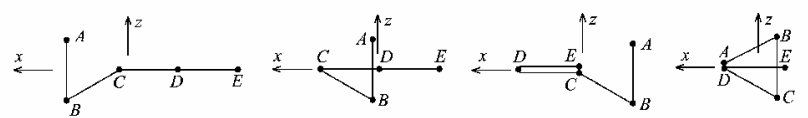

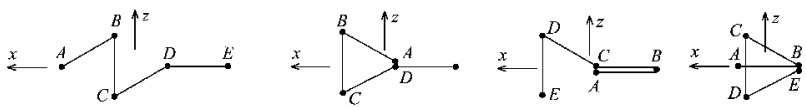

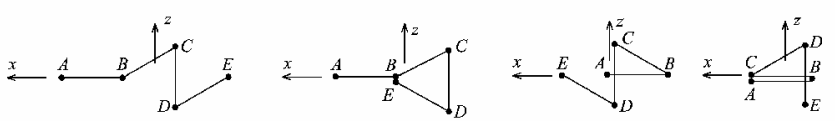

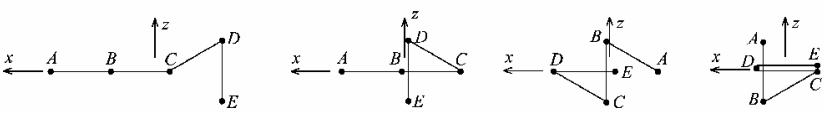

Fig. 4 Equilibria of a four-link chain (one vertical rod).

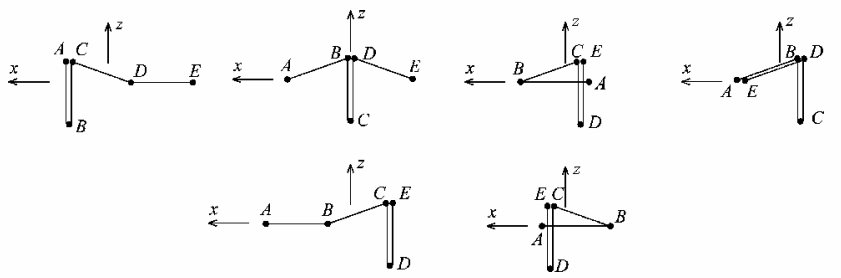

a)

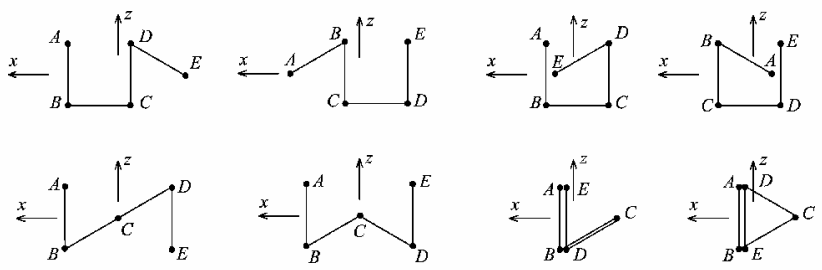

b)

Fig. 5 Equilibria of a four-link chain: a) one group of two adjacent rods and b) two separate vertical rods.
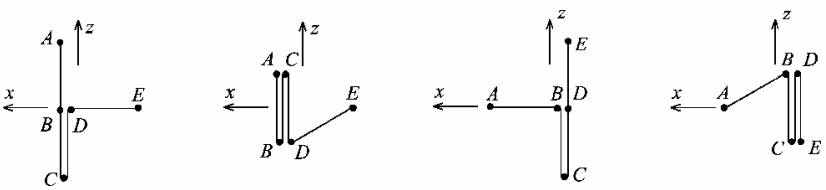

a)

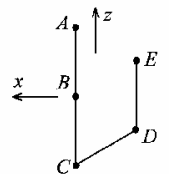<smiles>CC(C)(C)OC(C)(C)C</smiles>

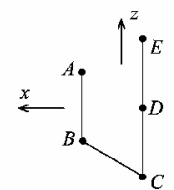

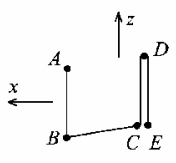

b)

Fig. 6 Equilibria of a four-link chain: a) one group of three vertical rods and b) two groups of vertical rods.

Equality $\sin \phi_{k}=0$ means that the $k$ th rod is vertical, and so we will study equilibrium configurations depending on the number of vertical rods and their positions in the chain.

\section{A. Horizontal Configurations}

We start the analysis considering the case when

$$
\prod_{k=1}^{n} \sin \phi_{k} \neq 0
$$

The equilibrium configurations correspond to the solutions of linear system

$$
\mathbb{B} z=0
$$

with nonzero determinant (22). So the unique solution of Eqs. (16) is

$$
z=0
$$

which means, in terms of angles $\phi_{k}$, that

$$
\cos \phi_{k}=0, \quad k=1, \ldots, n
$$

The orientation of the $k$ th member correspondsto $\phi_{k}= \pm \pi / 2$. Thus, in this case there are $2^{n}$ equilibria that exist for any lengths of the rods and masses of the satellites. All of them correspond to positions of the chain with all links pointed along the tangent to the orbit. Examples of such configurations are provided in Figs. 2a and $3 \mathrm{a}$.

\section{B. One Vertical Rod}

Consider now the case when there is only one number $k$ for which $\sin \phi_{k}=0$. The corresponding rod is parallel to the local vertical $O z$. Then $\phi_{k}=0$ or $\phi_{k}=\pi, \cos \phi_{k}= \pm 1$, and $\zeta_{k}=\zeta_{k}^{(0)}= \pm a_{k}$. The equations that describe the equilibrium configurations of the chain are

$$
\begin{array}{r}
b_{1,1} \zeta_{1}+\cdots+b_{1, k-1} \zeta_{k-1}+b_{1, k} \zeta_{k}^{(0)} \\
+b_{1, k+1} \zeta_{k+1}+\cdots+b_{1, n} \zeta_{n}=0 \\
b_{2,1} \zeta_{1}+\cdots+b_{2, k-1} \zeta_{k-1}+b_{2, k} \zeta_{k}^{(0)} \\
+b_{2, k+1} \zeta_{k+1}+\cdots+b_{2, n} \zeta_{n}=0
\end{array}
$$$$
\begin{aligned}
& b_{k-1,1} \zeta_{1}+\cdots+b_{k-1, k-1} \zeta_{k-1}+b_{k-1, k} \zeta_{k}^{(0)} \\
& \quad+b_{k-1, k+1} \zeta_{k+1}+\cdots+b_{k-1, n} \zeta_{n}=0 \\
& \zeta_{k}=\zeta_{k}^{(0)}
\end{aligned}
$$$$
b_{k+1,1} \zeta_{1}+\cdots+b_{k+1, k-1} \zeta_{k-1}+b_{k+1, k} \zeta_{k}^{(0)}
$$$$
+b_{k+1, k+1} \zeta_{k+1}+\cdots+b_{k+1, n} \zeta_{n}=0
$$$$
\begin{aligned}
& b_{n, 1} \zeta_{1}+\cdots+b_{n, k-1} \zeta_{k-1}+b_{n, k} \zeta_{k}^{(0)} \\
& +b_{n, k+1} \zeta_{k+1}+\cdots+b_{n, n} \zeta_{n}=0
\end{aligned}
$$

This system can be represented as

$$
\mathbb{D}_{J} z_{J}=-\boldsymbol{d}_{k J} \zeta_{k}^{(0)}, \quad J=\{k\}
$$

(Here the $k$ th row is omitted.)

Suppose first that $k=2, \ldots, n-1$. One can notice that it is possible to apply transformation (20) to all the lines of Eq. (32) except the $(k-1)$ th and $(k+1)$ th ones, and so this system is equivalent to

$$
\begin{gathered}
\zeta_{1}=0, \ldots, \zeta_{k-2}=0, \quad \zeta_{k}=\zeta_{k}^{(0)}, \quad \zeta_{k+2}=0, \ldots, \zeta_{n}=0 \\
b_{k-1, k-1} \zeta_{k-1}+b_{k-1, k} \zeta_{k}^{(0)}+b_{k-1, k+1} \zeta_{k+1}=0 \\
b_{k+1, k-1} \zeta_{k-1}+b_{k+1, k} \zeta_{k}^{(0)}+b_{k+1, k+1} \zeta_{k+1}=0
\end{gathered}
$$


Now we use the expressions for $b_{i, j}$ to determine $\zeta_{k-1}, \zeta_{k+1}$ and the respective angles of orientation of the rods:

$$
\begin{gathered}
\zeta_{k-1}=-\zeta_{k}^{(0)}\left[m_{k} /\left(m_{k-1}+m_{k}\right)\right] \\
\cos \phi_{k-1}=-\left(a_{k} / a_{k-1}\right)\left[m_{k} /\left(m_{k-1}+m_{k}\right)\right] \cos \phi_{k} \\
\zeta_{k+1}=-\zeta_{k}^{(0)}\left[m_{k-1} /\left(m_{k-1}+m_{k}\right)\right] \\
\cos \phi_{k+1}=-\left(a_{k} / a_{k+1}\right)\left[m_{k-1} /\left(m_{k-1}+m_{k}\right)\right] \cos \phi_{k}
\end{gathered}
$$

The condition of existence of this equilibrium is

$$
\begin{gathered}
\left(a_{k} / a_{k-1}\right)\left[m_{k} /\left(m_{k-1}+m_{k}\right)\right]<1 \\
\left(a_{k} / a_{k+1}\right)\left[m_{k-1} /\left(m_{k-1}+m_{k}\right)\right]<1
\end{gathered}
$$

[If $\left(a_{k} / a_{k-1}\right)\left[m_{k} /\left(m_{k-1}+m_{k}\right)\right]=1 \quad$ or $\quad\left(a_{k} / a_{k+1}\right)\left[m_{k-1} /\right.$ $\left.\left(m_{k-1}+m_{k}\right)\right]=1$, the $\operatorname{rod} a_{k-1}$ or $a_{k-1}$ is also aligned with the local vertical; we do not consider this case here.]

For $k=1$ the solution is given by the formulas

$$
\zeta_{2}=-\zeta_{1}^{(0)}\left[m_{0} /\left(m_{0}+m_{1}\right)\right]
$$

$\cos \phi_{2}=-\left(a_{1} / a_{2}\right)\left[m_{0} /\left(m_{0}+m_{1}\right)\right] \cos \phi_{1}$

$$
\text { if } \quad\left(a_{1} / a_{2}\right)\left[m_{0} /\left(m_{0}+m_{1}\right)\right]<1
$$

and for $k=n$ one gets

$$
\begin{gathered}
\zeta_{n-1}=-\zeta_{n}^{(0)}\left[m_{n} /\left(m_{n-1}+m_{n}\right)\right] \\
\cos \phi_{n-1}=-\left(a_{n} / a_{n-1}\right)\left[m_{n} /\left(m_{n-1}+m_{n}\right)\right] \cos \phi_{n} \\
\text { if } \quad\left(a_{n} / a_{n-1}\right)\left[m_{n} /\left(m_{n-1}+m_{n}\right)\right]<1
\end{gathered}
$$

Thus, the equilibrium configurations in this case are as follows: The $\operatorname{rod} a_{k}$ is aligned with the local vertical. The center of mass of the system of two points $A_{k-1}$ and $A_{k}$ connected by the rod $a_{k}$ belongs to the $x$ axis. All other rods of the chain except the direct neighbors of $a_{k}$ are also situated on the $x$ axis. (Examples are given by Figs. 2b, 2e, and 4.) Such equilibria exist if the lengths of the rods $a_{k-1}$ and $a_{k+1}$ (or of one of these rods if $k=1$ or $n$ ) suffice to allow this configuration:

$$
\begin{gathered}
a_{k-1}>a_{k}\left[m_{k} /\left(m_{k-1}+m_{k}\right)\right] \\
a_{k+1}>a_{k}\left[m_{k-1} /\left(m_{k-1}+m_{k}\right)\right]
\end{gathered}
$$

Because one can indicate two values of $\phi_{k}$ with the same cosine, the number of equilibria of this type does not exceed $n \cdot 2^{n}$.

\section{One Group of Vertical Rods}

Suppose that in system (16) $\sin \phi_{j}=0$ iff $j \in J=\{k+1, \ldots$, $k+r\}$. (This means that some group of $r$ adjacent rods is aligned with the local vertical.) Then $\cos \phi_{j}= \pm 1$, and $\zeta_{j}=\zeta_{j}^{(0)}= \pm a_{j}$ for $j \in J$. One can use property (20) to show that $\zeta_{j}=0$ for $j \notin J \bigcup\{k, k+r+1\}$. For $\zeta_{k}$ and $\zeta_{k+r+1}$ one arrives at the following system of equations:

$$
\begin{aligned}
& M_{0}^{k-1} M_{k}^{n} \zeta_{k}+M_{0}^{k-1} M_{k+1}^{n} \zeta_{k+1}^{(0)}+\cdots+M_{0}^{k-1} M_{k+r}^{n} \zeta_{k+r}^{(0)} \\
& \quad+M_{0}^{k-1} M_{k+r+1}^{n} \zeta_{k+r+1}=0 \\
& M_{0}^{k-1} M_{k+r+1}^{n} \zeta_{k}+M_{0}^{k} M_{k+r+1}^{n} \zeta_{k+1}^{(0)}+\cdots+M_{0}^{k+r-1} M_{k+r+1}^{n} \zeta_{k+r}^{(0)} \\
& \quad+M_{0}^{k+r} M_{k+r+1}^{n} \zeta_{k+r+1}=0
\end{aligned}
$$

Simplifying Eqs. (40), we get

$$
\begin{gathered}
\zeta_{k}+\zeta_{k+1}^{(0)}+\cdots+\zeta_{k+r}^{(0)}+\zeta_{k+r+1}=0 \\
M_{k}^{k+r} \zeta_{k}+M_{k+1}^{k+r} \zeta_{k+1}^{(0)}+\cdots+m_{k+r} \zeta_{k+r}^{(0)}=0
\end{gathered}
$$

and so

$$
\begin{gathered}
\zeta_{k}=-\left(M_{k+1}^{k+r} \zeta_{k+1}^{(0)}+\cdots+m_{k+r} \zeta_{k+r}^{(0)}\right) / M_{k}^{k+r} \\
\zeta_{k+r+1}=-\left(m_{k} \zeta_{k+1}^{(0)}+\cdots+M_{k}^{k+r-1} \zeta_{k+r}^{(0)}\right) / M_{k}^{k+r}
\end{gathered}
$$

Equalities (41) show that the center of mass of the group of vertical rods should lie on the local horizontal. There are no more than $2^{n}$ equilibrium configurations corresponding to solution (42). One of them exists if the lengths of the rods allow it:

$$
\begin{gathered}
\left|\left(M_{k+1}^{k+r} \zeta_{k+1}^{(0)}+\cdots+m_{k+r} \zeta_{k+r}^{(0)}\right) / M_{k}^{k+r}\right|<a_{k} \\
\left|\left(m_{k} \zeta_{k+1}^{(0)}+\cdots+M_{k}^{k+r-1} \zeta_{k+r}^{(0)}\right) / M_{k}^{k+r}\right|<a_{k+r+1}
\end{gathered}
$$

In case the group of vertical rods is located at one of the ends of the chain we get only one equation correspondingto the neighboring rod:

$$
m_{0} M_{r+1}^{n} \zeta_{1}^{(0)}+\cdots+M_{0}^{r-1} M_{r+1}^{n} \zeta_{r}^{(0)}+M_{0}^{r} M_{r+1}^{n} \zeta_{r+1}=0
$$

so that

$$
\zeta_{r+1}=-\left(m_{0} \zeta_{1}^{(0)}+\cdots+M_{0}^{r-1} \zeta_{r}^{(0)}\right) / M_{0}^{r}
$$

for $k=0$, and

$$
M_{0}^{k-1} M_{k}^{n} \zeta_{k}+M_{0}^{k-1} M_{k+1}^{n} \zeta_{k+1}^{(0)}+\cdots+M_{0}^{k-1} m_{n} \zeta_{n}^{(0)}
$$

and so

$$
\zeta_{k}=-\left(M_{k+1}^{n} \zeta_{k+1}^{(0)}+\cdots+m_{n} \zeta_{n}^{(0)}\right) / M_{k}^{n}
$$

for $k+r=n$. The respective equilibrium configurations possess the aforementionedpropertiesdescribedfor the case of an internalgroup of vertical rods (see Figs. 2c, 5a, and 6a).

System (16) also admits the solution

$$
\sin \phi_{j}=0, \quad j=1, \ldots, n
$$

which implies that the whole chain is situated on the local vertical. All the $2^{n}$ configurations are possible in this case (see Figs. $2 \mathrm{~d}$ and $3 b)$.

\section{Two or More Groups of Vertical Rods}

With no loss of generality, let us consider the case of two groups of vertical rods.

Suppose that $\sin \phi_{j}=0$ only for $j \in J=J_{1} \bigcup J_{2}, \quad J_{1}=$ $\left\{k_{1}+1, \ldots, k_{1}+r_{1}\right\}, \quad J_{2}=\left\{k_{2}+1, \ldots, k_{2}+r_{2}\right\} \quad$ with $k_{1}+r_{1}+$ $1 \leq k_{2}$. Then $\cos \phi_{j}= \pm 1$, and $\zeta_{j}=\zeta_{j}^{(0)}= \pm a_{j}$ for $j \in J$. Equations of equilibria are now

$$
\mathbb{D}_{J} z_{J}=-\left[\sum_{j \in J_{1}} \boldsymbol{d}_{j J} \zeta_{j}^{(0)}+\sum_{j \in J_{2}} \boldsymbol{d}_{j J} \zeta_{j}^{(0)}\right]
$$

Along with Eqs. (49), let us study the following two systems:

$$
\begin{aligned}
\mathbb{D}_{J} z_{J} & =-\sum_{j \in J_{1}} \boldsymbol{d}_{j J} \zeta_{j}^{(0)} \\
\mathbb{D}_{J_{1}} z_{J_{1}} & =-\sum_{j \in J_{1}} \boldsymbol{d}_{j J_{1}} \zeta_{j}^{(0)}
\end{aligned}
$$

System (50) was obtained from Eqs. (49) by putting $\zeta_{j}^{(0)}=0$ for $j \in J_{2}$. In accordance with Eq. (26), systems (49-51) have unique solutions. Obviously, system (51) is the system of equilibrium equations for a single group of vertical rods. It was examined before, and the solution implies $\zeta_{j}^{(0)}=0$ for $j \in\left\{1, \ldots, k_{1}-1, k_{1}+r_{1}+2, \ldots, k_{n}\right\}$, including $j \in J_{2}$. It means that the unique solution of system (50) is $\zeta_{j}=0$ for $j \notin J \bigcup\left\{k_{1}, k_{1}+r_{1}+1\right\}$ and $\zeta_{k_{1}}, \zeta_{k_{1}+r_{1}+1}$ are given by Eqs. (42) 
[or Eq. (45) if $k_{1}=0$ ] by putting $k \rightarrow k_{1}, r \rightarrow r_{1}$. Studying in the same way the systems

$$
\begin{aligned}
\mathbb{D}_{J} z_{J} & =-\sum_{j \in J_{2}} \boldsymbol{d}_{j J} \zeta_{j}^{(0)} \\
\mathbb{D}_{J_{2}} z_{J_{2}} & =-\sum_{j \in J_{2}} \boldsymbol{d}_{j J_{2}} \zeta_{j}^{(0)}
\end{aligned}
$$

one arrives at the conclusion that the solution of Eq. (53) is $\zeta_{j}=0$ for $j \notin J_{2} \bigcup\left\{k_{2}, k_{2}+r_{2}+1\right\}$ and $\zeta_{k_{2}}, \zeta_{k_{2}+r_{2}+1}$ are calculated by Eqs. (42) [or Eq. (47) if $k_{2}+r_{2}=n$ ) with substitution $k \rightarrow k_{2}, r \rightarrow r_{2}$. So the solution of Eq. (52) is $\zeta_{j}=0$ for $j \notin J \bigcup\left\{k_{2}, k_{2}+r_{2}+1\right\}$ with the same values of $\zeta_{k_{2}}$ and $\zeta_{k_{2}+r_{2}+1}$.

To get the solutions of the initial system (49), one has to sum the solutions of Eqs. (50) and (52). Thus the solutions of system (16) that correspond to two (or more) groups of vertical rods can be found by the superposition of solutions obtained for each group of vertical rods separately.

The respective equilibria configurations represent two groups of vertical rods with their centers of mass situated on the $x$ axis. For these rows $\zeta_{j}=\zeta_{j}^{(0)}= \pm a_{j}$ for $j \in J$. If these groups have no common neighbor, the orientations of the neighbor rods are described by Eqs. (42) [or Eqs. (45) and (47) if it is the case]. These rods connect the vertical groups with the rest of the chain that is oriented horizontally.

If $k_{1}+r_{1}+1=k_{2}$, that is, the vertical groups have a common neighbor, its orientation is described by the relation

$$
\tilde{\zeta}_{k_{2}}=\zeta_{k_{1}+r_{1}+1}+\zeta_{k_{2}}
$$

where $\zeta_{k_{1}+r_{1}+1}$ is given by Eqs. (42) with the substitution $k \rightarrow k_{1}, r \rightarrow r_{1}$, and $\zeta_{k_{2}}$ is given with the substitution $k \rightarrow k_{2}, r \rightarrow r_{2}$. The only difference with the preceding case is that the common neighbor of the vertical groups should be oriented so as to guarantee that their centers of mass still belong to the local horizontal (see Figs. 2f, 5b, and 6b).

Obviously, the same procedure can be applied to study equilibrium configurations with three or more groups of vertical rods. This type of equilibrium exists if the inclined rods are long enough.

\section{E. Number of Equilibria}

The total number of equilibria in the plane of the orbit does not exceed product of the number of possible choices of vertically oriented rods and the number of respective configurations of the chain:

$$
N \leq 2^{n} \cdot 2^{n}=2^{2 n}
$$

The exact number of equilibria can be determined by taking into account the lengths of the links and the masses of the points $A_{0}, \ldots, A_{n}$.

\section{Examples}

We consider two examples to illustrate the general results obtained in Sec. V. The first is a three-link chain with different members; the second one is a four-link chain with equal masses of the satellites and lengths of the connecting rods. Equilibria of these structures are schematically represented by Figs. 2-6. Even in these cases of relatively few links, we have too many configurations to depict them all, and so we indicate the type of symmetry that permits one to obtain the remaining equilibria.

\section{A. Three-Link Chain}

Figure 2 illustrates the results of analysis of equilibrium configurations for a three-link chain that consists of four material points $A, B, C$, and $D$. The masses of the points are respectively $m_{A}=m$, $m_{B}=2 m, m_{C}=4 m$, and $m_{D}=8 m$. The connecting rods have the lengths $l_{A B}=l, l_{B C}=2 l$, and $l_{C D}=4 l$. Figure 2 a shows four of the existing $2^{3}=8$ horizontal configurations; the other four are symmetric with respect to the $z$ axis. Figure $2 \mathrm{~b}$ represents two essentially different configurations with one vertical rod; the other six equilibria are obtained by reflections with respect to $x$ and/or $z$ axes. For this chain, there are no equilibria where either $B C$ or $C D$ is the only vertical rod; the reason is illustrated in Fig. 2e. In this configuration, $z_{B}=2 l_{B C} / 3=4 l / 3$, but point $A$ should lie on the $x$ axis. This is possible only if $l_{A B}>4 l / 3$, which is not the case. All essentially different existing equilibria with a group of two vertical rods are shown in Fig. 2c. (The remaining nine configurations are obtained by symmetries with respect to $x$ and/or $z$ axis.) For this chain, there are no equilibria with two groups of vertical rods. As shown in Fig. 2e, the existence of such equilibria would imply that $l_{B C}>z_{C}-z_{B}=2 l_{C D} / 3-l_{A B} / 3=7 l / 3$. In Fig. $2 \mathrm{~d}$, one can see four existing vertical configurations; the other four are symmetric with respect to the $x$ axis. In this case there exist 36 equilibrium configurations of the chain. [The upper bound of Eq. (55) is $2^{6}=64$.]

\section{B. Four-Link Chain}

Now let us consider equilibria of a four-link chain in the plane of a circular orbit. To simplify the calculations we choose a system of five material points $A, B, C, D$, and $E$ of equal masses. The lengths of the links are also equal. Figures 3-6 represent schematically five groups of possible equilibria.Figure 3 shows all essentially different equilibria, where the rods are either all horizontal or all vertical; the others are symmetric with respect to $z$ or $x$ axis correspondingly. Figures 4-6 show schemes of existing equilibria where orientations of rods may differ; the rest of them are symmetric with respect to $x$ and/or $z$ axis. The total number of equilibrium configurations is $N=192$. The difference between the estimate (55) and the actual quantity $2^{8}-192=64$ originates in the specific dimensions of the chain. For example, a unique group of two vertical rods aligned with the same direction proves impossible, because the rod adjacent to this group would have to be vertical as well. Consequently, we deal with the case of three adjacent vertical rods (two of them pointing in the same direction, and the third in the opposite one), which are represented in Fig. 6.

\section{Conclusions}

We study equilibria of a multibody connected system within the framework of the model of an $n$-link chain. All possible configurations in the plane of a circular orbit are described. It is shown that each rod of the chain can occupy one of the following positions:

1) It can be directed along the local horizontal $O x$ (tangent to the orbit).

2) It can be a member of a group of $k$ consequent vertical rods and is the center of mass of this group situated on the axis $O x$.

3) An oblique orientation is possible if the rod joins either two vertical groups of rods or the end of a vertical group with the axis $O x$.

Each of these configurationsexists when the lengths of the oblique rods (if there are any) allow it. The total number of equilibria does not exceed $2^{2 n}$.

For the particular case of a two-member link the results obtained coincide with those of Refs. 5-9.

\section{Acknowledgments}

This work is supported by the Portuguese Foundation for Science and Technology. We express our gratitude to A. K. Misra for interesting discussions of the problem.

\section{References}

${ }^{1}$ Keshmiri, M., Misra, A. K., and Modi, V. J., "General Formulation for $N$-Body Tethered Satellite System Dynamics," Journal of Guidance, Control, and Dynamics, Vol. 19, No. 1, 1996, pp. 75-83.

${ }^{2}$ Kalantzis, S., Modi, V. J., Padhan, S., and Misra, A. K., “Order- $N$ Formulation and Dynamics of Multibody Tethered Systems," Journal of Guidance, Control, and Dynamics, Vol. 21, No. 2, 1998, pp. 277-285.

${ }^{3}$ Chen, Y., Modi, V. J., De Silva, C. W., and Misra, A. K., "On the Performance of Variable Geometry Manipulators: Part I-Three Dimensional Order N Formulation," Acta Astronautica, Vol. 49, No. 1, 2001, pp. $1-12$.

${ }^{4}$ Nagata, T., Modi, V. J., and Matsuo, H., "Dynamics and Control of Flexible Multibody Systems-Part I: General Formulation with an Order N Forward Dynamics," Acta Astronautica, Vol. 49, No. 11, 2001, pp. 581-594. 
${ }^{5}$ Sarychev, V. A., "Positions of Relative Equilibriumfor Two Bodies Connected by a Spherical Hinge in a Circular Orbit," Cosmic Research, Vol. 5, No. 3, 1967, pp. 360-364.

${ }^{6}$ Lorenzini, E. C., "A Three-Mass Tethered System for Micro- $g$ /Variable$g$ Applications," Journal of Guidance, Control, and Dynamics, Vol. 10, No. 3, 1987, pp. 242-249.

${ }^{7}$ Misra, A. K., Amier, Z, and Modi, V. J., "Attitude Dynamics of Three-Body Tethered Systems," Acta Astronautica, Vol. 17, No. 10, 1988, pp. 1059-1068.

${ }^{8}$ Sarychev, V. A., "Equilibria of a Double Pendulum in a Circular Orbit,"
Acta Astronautica, Vol. 44, No. 1, 1999, pp. 63-65.

${ }^{9}$ Misra, A. K., "Equilibrium Configurations of Tethered Three-Body Systems and Their Stability," Spaceflight Mechanics 2001, Advances in the Astronautical Sciences, Vol. 108, Pt. 2, Univelt, San Diego, CA, 2001, pp. $1757-1773$.

${ }^{10}$ Breakwell, J. V., "Stability of an Orbiting Ring," Journal of Guidance and Control, Vol. 4, No. 2, 1981, pp. 197-200.

${ }^{11}$ Beletsky, V. V., and Levin, E. M., "Stability of a Ring of Connected Satellites," Acta Astronautica, Vol. 12, No. 10, 1985, pp. $765-769$. 